\title{
Correction to: The atypical RhoGTPase RhoE/Rnd3 is a key molecule to acquire a neuroprotective phenotype in microglia
}

Veronika E. Neubrand", Irene Forte-Lago, Marta Caro and Mario Delgado*

\section{Correction to: J Neuroinflammation} https://doi.org/10.1186/s12974-018-1386-z

In the version of this article that was originally published [1]; some information in the "Authors' contributions" section was omitted. This has now been corrected in this Correction note.

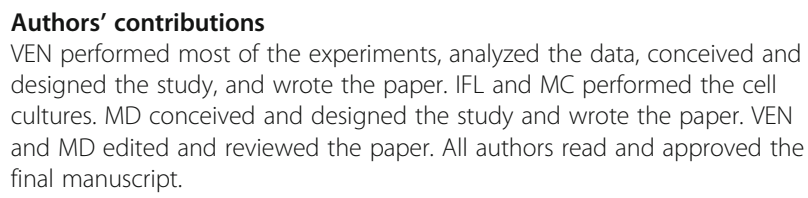

Published online: 12 February 2019

\section{Reference}

1. Neubrand VE, et al. The atypical RhoGTPase RhoE/Rnd3 is a key molecule to

acquire a neuroprotective phenotype in microglia. J Neuroinflammation.

2018;15:343. https://doi.org/10.1186/s12974-018-1386-z.

\footnotetext{
* Correspondence: veronika.neubrand@gmail.com; mdelgado@ipb.csic.es Instituto de Parasitología y Biomedicina López-Neyra, IPBLN-CSIC, Avd. Conocimiento 17, PTS Granada, 18016 Granada, Spain
} 